\title{
EDITORIALE
}

\section{Uno strumento di qualità}

\author{
An instrument of quality
}

Nella Carta della Qualità in Medicina Interna, elaborata dalla Federazione delle Associazioni dei Dirigenti Ospedalieri Internisti (FADOI) insieme all'Associazione Nazionale Infermieri Medicina Ospedaliera (ANIMO) e a Cittadinanza Attiva, si legge che il paziente, al momento della dimissione, "deve ottenere tutte le informazioni necessarie ad affrontare al meglio il rientro a casa, secondo modalità che assicurino una adeguata continuità assistenziale. [.. .] Il paziente riceve una dettagliata scheda di dimissione che riporta la diagnosi, la terapia praticata, il decorso, le indicazioni mediche e infermieristiche e i comportamenti da adottare".

In queste frasi è riportata una parola, diagnosi, alla quale non viene attribuito alcun rilievo particolare in un elenco in cui, in quel particolare contesto, non doveva certamente averne; pur tuttavia sarebbe stato probabilmente opportuno marcare con forza quell'aspetto dell'atto medico che sembra aver perso, nel tempo, gran parte del suo valore.

Ci troviamo di fronte alla crisi della diagnosi o a ciò che essa sottende, cioè alla crisi del ragionamento clinico? Nella diagnosi si racchiude il percorso che il paziente ha compiuto durante la degenza in base al programma ideato dal medico. Essa diventa l'istantanea di quanto fin lì ha vissuto, ma non è, in verità, un fotogramma fisso: è un divenire di immagini nelle quali si intravede il futuro del paziente. E il futuro non si riferisce soltanto all'aspetto clinico, ma anche a quello della vita affettiva, dei desideri, delle rinunce, delle privazioni, delle aspirazioni. Si può, infatti, separare la malattia, con i comportamenti che essa richiede, dal quotidiano sentire dell'uomo? E allora, affinché l'ombra dell'una non stenda un mantello oppressivo, l'opera del medico ha l'obiettivo di trovare il giusto equilibrio, il punto di chiarezza di una vita che deve essere pienamente vissuta.

Nel momento in cui si espone, in poche righe, l'elenco delle malattie di quella persona, devono manifestarsi in qualche modo la cultura e l'esperienza di chi scrive, in una visione globale che colga tutti i possibili aspetti.

L'uomo si affida al medico, è nelle sue mani e in quelle mani è racchiusa la sua vita. Il medico non deve dimenticare questo totale affidamento e, nel suo agire, non deve esserne schiacciato; al contrario, deve far trasparire la delicatezza di quel rapporto così totalizzante.

La diagnosi segna il limite oltre il quale si carica su quella persona il peso di un giudizio che trascende il presente e si proietta nel futuro.

Per tutti questi motivi la costruzione del processo diagnostico assume caratteristiche importanti e costituisce la cifra scientifica e antropologica della Medicina Interna. È proprio nel tratto "caleidoscopico" della diagnosi internistica il suo aspetto peculiare, nel quale appare la pluralità delle patologie arricchite, come afferma Vito Cagli, dalla diagnosi anatomica (imaging, biopsie), dalla diagnosi integrata (paziente, ambiente, società) e dalla diagnosi fisiopatologica (laboratorio).

Nella lettera di dimissione che il medico consegna al malato la diagnosi deve essere posta in tutta evidenza, nella forma più trasparente e con le espressioni più comprensibili. Essa rappresenta l'atto conclusivo di un'esperienza e di un ragionamento che il medico sottoscrive impegnandosi professionalmente e moralmente.

Il tempo della degenza è stato contratto al massimo, tutto è stato fatto il più velocemente possibile, ma nell'ora della diagnosi si deve recuperare la dimensione della lentezza, nella quale si dilata il tempo della riflessione critica che conduce alla diagnosi armonica e completa.

Nel campo della Medicina Interna, in cui il problema della complessità e criticità diverse si influenzano e si alimentano a vicenda in quella singola persona, lo sforzo di sintesi diagnostica diventa maggiore e assume un valore più qualitativo. Non si tratta, come potrebbe apparire, di mero esercizio culturale o scientifico, ma della verifica di una gestione clinica coerente sia con le migliori e maggiori evidenze sia con i bisogni della persona.

Francesco D'Amore Tesoriere nazionale FADOI E-mail: dott_damore@libero.it 